\title{
NONLINEAR POTENTIALS IN FUNCTION SPACES
}

\author{
MURALI RAO AND ZORAN VONDRAČEK ${ }^{1) 2}$
}

\begin{abstract}
We introduce a framework for a nonlinear potential theory without a kernel on a reflexive, strictly convex and smooth Banach space of functions. Nonlinear potentials are defined as images of nonnegative continuous linear functionals on that space under the duality mapping. We study potentials and reduced functions by using a variant of the Gauss-Frostman quadratic functional. The framework allows a development of other main concepts of nonlinear potential theory such as capacities, equilibrium potentials and measures of finite energy.
\end{abstract}

\section{§1. Introduction}

The goal of this paper is to present a fairly general setting which allows a development of basic concepts of nonlinear potential theory. This setting provides a unified approach to several aspects of nonlinear potential theory with kernel, as well as some kernel free potential theory. The concepts that can be developed include capacities of sets and functions, nonlinear potentials, equilibrium potentials, reduced functions, balayage, and measures of finite energy.

The framework of our approach is a reflexive, strictly convex and smooth Banach space of functions satisfying two additional hypotheses. Nonlinear potential theory in function spaces has been the subject of research in several papers during seventies (e.g., [7], [12], [19]). The goal was to extend the Dirichlet space theory to the nonlinear setting. This was achieved under various hypotheses. The common hypothesis was that the underlying function space is a Banach space with a vector lattice structure.

Almost at the same time, a different type of nonlinear potential theory began to take shape in the works of Fuglede, Meyers, and Havin and

Received July 13, 2000.

2000 Mathematics Subject Classification: 31C15, 31C45, 46B10, 46Exx, 46E35, $47 \mathrm{H} 07$.

${ }^{1)}$ This work was done while the author was visiting Department of Mathematics, University of Florida. The support and hospitality is gratefully acknowledged.

${ }^{2)}$ Supported in part by the MZT Grant 037008 of the Republic of Croatia. 
Maz'ya ([13], [21], [16]). This approach was based on a kernel, and has been fully developed in the book by Adams and Hedberg ([1], 1996). Besides the usual $L^{p}$-theories (which by use of $\alpha$-Bessel kernel lead to Sobolev spaces $\left.H^{\alpha, p}\right)$, variants were developed to accomodate function spaces like Besov spaces and Lizorkin-Triebel spaces (see [1, pp. 104-108], and in a somewhat different form [22]). Another variant, developed by Aïssaoui and Benkirane in [2] and [3], is to replace the $L^{p}$-space by a nice Orlicz space. We also mention the paper by Kazumi and Shikegawa, [18], where a nonlinear potential theory based on a contraction semigroup on $L^{p}$ was developed.

The usual setting for $L^{p}$-theories with kernel is roughly as follows: Let $X$ be a Hausdorff topological space (most often locally compact), and let $Y$ be a measureable space equipped with a positive measure $\nu$. For $1<p<$ $\infty$, let $L^{p}(Y, \nu)$ denote the space of $p$-integrable functions (or equivalence classes of functions) on $Y$. A kernel on $X \times Y$ is a function $v: X \times Y \rightarrow$ $[0, \infty]$ satisfying certain regularity assumptions which depend on the precise settings. Potentials of functions are defined as follows: For a nonnegative function $f$ on $Y$, or for $f \in L^{p}(Y, \nu)$, let

$$
V f(x):=\int_{Y} v(x, y) f(y) \nu(d y) .
$$

A norm can be introduced on the linear space of such potentials of functions providing it with the Banach space strucure. On the other hand, it is rarely the case that such a space is a lattice.

We propose a framework of a nonlinear potential theory in a function space which is broad enough to accomodate basic features of nonlinear potential theories with a kernel. The formulation of the theory is in the spirit of Dirichlet space theory, and is also related to the work of Aronszajn and Smith in [5]. In studying equilibrium potentials and reduced functions we minimize convex functions of the norm over closed convex sets. This invites variational methods and one is inevitably led to the concept of duality mapping. A duality mapping on the dual of the underlying function space is the key to our approach. We define (nonlinear) potentials to be images, under the duality mapping, of nonnegative continuous linear functionals on the function space. Similar definition also appears in [7], [12] and [19]. Our definition of a nonlinear potential generalizes the definition of nonlinear potentials in potential theories with a kernel (see Section 5, Example A). The basic assumption we impose is that the duality mapping (on the dual) is nonnegative, ensuring that potentials are nonnegative functions. 
The concept of the potential is the cornerstone of our approach, and besides giving several equivalent definitions and convergence properties, we also point out the usefulness of potentials in applications. These ideas and applications will be expounded elsewhere. We also pay a close attention to reduced functions, a concept very important in linear potential theory, but not much studied in nonlinear settings. Instead of employing the usual minimax theorem for studying the dual representation of the reduced function, we propose a different method based on a variant of the Gauss-Frostman quadratic functional. A neat treatment of reduced functions following from this approach is the second main feature of this work.

Let us point out that the proposed framework (with additional topological and analytical hypotheses) allows a development of other basic ideas and results of a nonlinear potential theory such as capacities of sets and functions, quasicontinuous modifications of functions, equilibrium potentials and measures of finite energy. We have obtained the usual results folowing the methods from [11], [14], [15] and [18], as well as the modified Gauss-Frostman method for the dual representation of the capacity. In particular, under appropriate conditions, positive linear functionals are identified with measures of finite energy. To stay concentrated on what is new in our approach, we decided to omit these results from the present paper, and leave them to future extended notes. Moreover, leaving aside topological and analytical hypotheses shows clearly that certain potentialtheoretic concepts can be developed in a purely functional-analytic setting. In order to show the versatility of our approach, we discuss examples of function spaces that fit in our framework.

Organization of the paper: In the next section we introduce the setting and hypotheses. Potentials are studied in Section 3, and reduced functions in Section 4. In the last section we present several examples.

\section{$\S 2$. Setting and hypotheses}

Let $X$ be a nonempty set, and let $\mathcal{N}$ be an ideal of subsets of $X$. More precisely,

(i) If $N \in \mathcal{N}$ and $M \subset N$, then $M \in \mathcal{N}$, and

(ii) If $N_{k} \in \mathcal{N}$ for $k \in \mathbb{N}$, then $\bigcup_{k=1}^{\infty} N_{k} \in \mathcal{N}$.

We say that a property holds $\mathcal{N}$ almost everywhere $(\mathcal{N}$-a.e. $)$ if it holds outside of a set from $\mathcal{N}$.

Let $\mathcal{F}$ be a vector space of $\mathcal{N}$-a.e. defined functions taking values in $[-\infty,+\infty]$ such that for every $u \in \mathcal{F},\{|u|=+\infty\} \in \mathcal{N}$. We assume that 
there is a seminorm \|\| on $\mathcal{F}$ such that $\|u\|=0$ for $u \in \mathcal{F}$, implies that $u=0 \mathcal{N}$-a.e. As it is customary, we identify functions that are equal $\mathcal{N}$ a.e., and denote by the same letter $\mathcal{F}$ the vector space of equivalence classes. Then $(\mathcal{F},\|\|)$ becomes a normed space. There is a natural order on $\mathcal{F}$ : for $u, v \in \mathcal{F}, v \geq u$ (as equivalence classes) if $v \geq u \mathcal{N}$-a.e. (as functions).

In most applications $\mathcal{N}$ will be the ideal of sets of measure zero for the underlying measure, or the ideal of quasi-null sets. In the latter situation, one would deal with quasicontinuous modifications of functions from $\mathcal{F}$.

Let $\mathcal{F}^{*}$ denote the dual space of $\mathcal{F}$ with the dual norm \|\| . The duality pairing between $\phi \in \mathcal{F}^{*}$ and $u \in \mathcal{F}$ will be denoted by $\langle\phi, u\rangle$. We will assume that $(\mathcal{F},\|\|)$ satisfies the following hypotheses:

Hypothesis $(\mathbf{F})$ ON $(\mathcal{F},\|\|)$.

(i) $\mathcal{F}$ is a reflexive Banach space,

(ii) $\mathcal{F}$ is strictly convex, and

(iii) $\mathcal{F}$ is smooth.

Let us recall that the normed space $\mathcal{F}$ is strictly convex if for every $u, v \in \mathcal{F}$ such that $u \neq v$ and $\|u\|=\|v\|=1$, it follows that $\|u+v\|<2$. The space $\mathcal{F}$ is smooth if for every $u \in \mathcal{F}, u \neq 0$, there exists a unique $\phi \in \mathcal{F}^{*}$ such that $\langle\phi, u\rangle=\|u\|$ and $\|\phi\|=1$. It is well known that $\mathcal{F}$ is smooth if and only if the norm \|\| is Gateaux differentiable on $\mathcal{F} \backslash\{0\}$ (e.g. [8, Theorem I. 3.5]). Moreover, since $\mathcal{F}$ is reflexive, $\mathcal{F}$ is smooth (respectively strictly convex) if and only if $\mathcal{F}^{*}$ is strictly convex (respectively smooth) ([8, Corollary II. 1.4]). Therefore, by our hypotheses on $\mathcal{F}$, the dual space $\mathcal{F}^{*}$ is also strictly convex and smooth.

We will also need a hypothesis that relates convergence in $\mathcal{F}$ with the pointwise convergence of functions:

Hypothesis $(\mathbf{C})$. If $\left(u_{n}, n \in \mathbb{N}\right)$ is a sequence in $\mathcal{F}$ such that $\lim _{n \rightarrow \infty} u_{n}=u$ in $\mathcal{F}$, then there exists a subsequence $\left(n_{k}, k \in \mathbb{N}\right)$ such that $\lim _{k \rightarrow \infty} u_{n_{k}}=u \mathcal{N}$-a.e.

Let us recall several facts about the duality mapping (due to Asplund [6]; see also [8, Corollary I. 4.5]). Let $\gamma:[0, \infty) \rightarrow[0, \infty)$ be a continuous and strictly increasing function such that $\gamma(0)=0$ and $\lim _{t \rightarrow \infty} \gamma(t)=+\infty$. Such a function $\gamma$ will be called a weight function. Let $\Gamma:[0, \infty) \rightarrow[0, \infty)$ be defined by

$$
\Gamma(t)=\int_{0}^{t} \gamma(s) d s
$$


Then $\Gamma$ is a convex function (and clearly strictly increasing). Since $\mathcal{F}$ is smooth, there exists a (single valued) duality mapping $J: \mathcal{F} \rightarrow \mathcal{F}^{*}$ characterized by

$$
\langle J u, u\rangle=\|J u\|\|u\| \text { and }\|J u\|=\gamma(\|u\|) .
$$

Moreover,

$$
\langle J u, v\rangle=\frac{d}{d t} \Gamma(\|u+t v\|)_{\mid t=0} \quad \text { for any } u, v \in \mathcal{F} .
$$

Let us mention that $J: \mathcal{F} \rightarrow \mathcal{F}^{*}$ is a nonlinear operator. The duality mapping does not essentially depend on the choice of the weight function. If $\widehat{\gamma}$ is another weight function with the corresponding duality mapping $\widehat{J}$, then

$$
\widehat{J} u=\frac{\widehat{\gamma}(\|u\|)}{\gamma(\|u\|)} J u, \quad \text { for any } u \neq 0 .
$$

The duality mapping corresponding to the weight function $\gamma(t)=t$ is called the normalized duality mapping. In that case, for $u \neq 0, J u$ is the gradient of the norm at $u$.

Let $\gamma^{*}=\gamma^{-1}$ be the inverse function of $\gamma$, and define

$$
\Gamma^{*}(t)=\int_{0}^{t} \gamma^{*}(s) d s .
$$

Let $U: \mathcal{F}^{*} \rightarrow \mathcal{F}$ be the duality mapping of weight $\gamma^{*}$ (which is single valued due to the fact that $\mathcal{F}^{*}$ is also smooth). Then both $J$ and $U$ are 1-1 and onto, and moreover $U=J^{-1}$ (e.g. [8, Corollary II. 3.5]). The dual form of (2.2) reads

$$
\langle\psi, U \phi\rangle=\frac{d}{d t} \Gamma^{*}(\|\phi+t \psi\|)_{\mid t=0} \quad \text { for any } \phi, \psi \in \mathcal{F}^{*} .
$$

Let $\mathcal{F}_{+}$denote the convex cone of nonnegative elements from $\mathcal{F}: u \in \mathcal{F}_{+}$ if $u \geq 0$ (i.e., $u \geq 0 \mathcal{N}$-a.e. as a function). Let $\mathcal{F}_{+}^{*}$ denote the dual cone of $\mathcal{F}_{+}$. Thus, $\phi \in \mathcal{F}_{+}^{*}$ if $\langle\phi, u\rangle \geq 0$ for every $u \in \mathcal{F}_{+}$. The following simple result is fundamental.

TheOREM 2.1. (i) Let $\mathcal{K} \subset \mathcal{F}$ be a closed, convex set. Then there exists a unique $u_{0} \in \mathcal{K}$ such that

$$
\left\|u_{0}\right\|=\min \{\|u\|: u \in \mathcal{K}\}
$$

(ii) If $\mathcal{K}$ has the property that $u \in \mathcal{K}, v \in \mathcal{F}_{+}$imply that $u+v \in \mathcal{K}$, then $J u_{0} \in \mathcal{F}_{+}^{*}$. 
Proof. (i) The existence of $u_{0}$ follows from the reflexivity of $\mathcal{F}$ by Mazur's lemma. The uniqueness follows from strict convexity of the norm. (ii) Note that since $\Gamma$ is strictly increasing, it holds that

$$
\Gamma\left(\left\|u_{0}\right\|\right)=\min \{\Gamma(\|u\|): u \in \mathcal{K}\}
$$

Let $v \geq 0$. Then $u_{0}+t v \in \mathcal{K}$ for every $t \geq 0$. Hence $\Gamma\left(\left\|u_{0}+t v\right\|\right) \geq \Gamma\left(\left\|u_{0}\right\|\right)$ for every $t \geq 0$, implying that

$$
0 \leq \frac{d}{d t} \Gamma\left(\left\|u_{0}+t v\right\|\right)_{\mid t=0}=(2.2)=\left\langle J u_{0}, v\right\rangle .
$$

Since this holds for every $v \geq 0$, it follows that $J u_{0} \in \mathcal{F}_{+}^{*}$.

For a function $u \in \mathcal{F}$ let $u^{+}=\max \{u, 0\}$. We introduce now a new hypothesis on $\mathcal{F}$ which is crucial:

Hypothesis $\left(\mathbf{D}^{+}\right)$. For every $u \in \mathcal{F}$ there exists $v \in \mathcal{F}$ such that $u^{+} \leq v$ and $\|v\| \leq\|u\|$.

The following result shows that $\left(\mathbf{D}^{+}\right)$is the correct hypothesis if one wants potentials to be nonnegative functions.

Proposition 2.2. The following are equivalent:

(i) Hypothesis $\left(\mathbf{D}^{+}\right)$holds true.

(ii) The duality mapping $U: \mathcal{F}^{*} \rightarrow \mathcal{F}$ is nonnegative in the sense that if $\phi \in \mathcal{F}_{+}^{*}$, then $U \phi \in \mathcal{F}_{+}$.

Proof. (i) $\Rightarrow$ (ii): Let $\phi \in \mathcal{F}_{+}^{*} \backslash\{0\}$, and let $u=U \phi$. Let us consider the set

$$
\mathcal{K}_{u}:=\{v \in \mathcal{F}: v \geq u\} .
$$

Then $\mathcal{K}_{u}$ is convex, closed and satisfies the assumption from Theorem 2.1 (ii) (note that closedness follows from hypothesis $(\mathbf{C})$ ). Let

$$
\left\|u_{0}\right\|=\min \left\{\|v\|: v \in \mathcal{K}_{u}\right\}
$$

By 2.1 (ii), $J u_{0} \geq 0$ in $\mathcal{F}^{*}$. By $\left(\mathbf{D}^{+}\right)$, there exists $v \in \mathcal{F}$ such that $v \geq u_{0}^{+} \geq$ 0 and $\|v\| \leq\left\|u_{0}\right\|$. Therefore, $v \geq u_{0} \geq u$, hence $v \in \mathcal{K}_{u}$. Since $\|v\| \leq\left\|u_{0}\right\|$, by uniqueness $v=u_{0}$. Therefore, $u_{0} \geq 0$.

We show now that $u=u_{0}$ which proves the claim. Note that $J u=$ $J(U \phi)=\phi \geq 0$. Since $u_{0}-u \geq 0$, it follows that $0 \leq\left\langle J u, u_{0}-u\right\rangle$, 
i.e., $\langle J u, u\rangle \leq\left\langle J u, u_{0}\right\rangle$. From $(2.1),\langle J u, u\rangle=\|J u\|\|u\|$, while $\left\langle J u, u_{0}\right\rangle \leq$ $\|J u\|\left\|u_{0}\right\|$. Therefore, $\|u\| \leq\left\|u_{0}\right\|$. Since $\left\|u_{0}\right\| \leq\|u\|$ by definition, uniqueness and the fact that $u \in \mathcal{K}_{u}$ imply that $u=u_{0}$.

(ii) $\Rightarrow$ (i): Let $u \in \mathcal{F}$, and let $\left\|u_{0}\right\|=\min \{\|v\|: v \geq u\}$. Then $u_{0} \geq u$, $\left\|u_{0}\right\| \leq\|u\|$, and by Theorem 2.1 (ii), $J u_{0} \geq 0$. By the assumption on $U$, $u_{0}=U\left(J u_{0}\right) \geq 0$. Thus, $u_{0} \geq u$ and $u_{0} \geq 0$, implying that $u_{0} \geq u^{+}$. Since $\left\|u_{0}\right\| \leq\|u\|,\left(\mathbf{D}^{+}\right)$holds true.

It is worth pointing out that $J: \mathcal{F} \rightarrow \mathcal{F}^{*}$ need not be nonnegative. Let us note that nonnegativity of $U$ may be regarded as a replacement for the concept of obtuse cones studied in [9]. A Dirichlet space theory view on $\left(\mathbf{D}^{+}\right)$reveals it as a weak form of the contraction principle. A slightly stronger form would be the following

Hypothesis (D). For every $u \in \mathcal{F}$ there exists $v \in \mathcal{F}$ such that $|u| \leq v$ and $\|v\| \leq\|u\|$.

It is clear that $(\mathbf{D})$ implies $\left(\mathbf{D}^{+}\right)$. On the other hand, if $\left(\mathbf{D}^{+}\right)$holds true, then for given $u \in \mathcal{F}$, there exists $v \in \mathcal{F}$ such that $|u| \leq v$, but $\|v\| \leq 2\|u\|$. Hypothesis (D) is needed for a development of a satisfactory concept of capacity of sets and functions. We will have no need of it in the present paper. From now on, we assume that hypotheses $(\mathbf{F}),(\mathbf{C})$ and $\left(\mathbf{D}^{+}\right)$hold true.

At the end of this section, let us introduce a variant of the GaussFrostman quadratic functional. For $u \in \mathcal{F}$ let $Q=Q_{u}: \mathcal{F}^{*} \rightarrow \mathbb{R}$ be the mapping defined by

$$
Q(\phi):=\Gamma^{*}(\|\phi\|)-\langle\phi, u\rangle
$$

Minimization of the functional $Q$ over various subsets of functionals in $\mathcal{F}^{*}$ will be one of our main tools in studying potentials and reduced functions. Note that $Q$ is a weak-* lower semicontinuous function on $\mathcal{F}^{*}$. Indeed, this follows from the well-known fact that $\phi \mapsto\|\phi\|$ is weak-* 1.s.c., from continuity of $\Gamma^{*}$, and weak-* continuity of the mapping $\phi \mapsto\langle\phi, u\rangle$.

\section{§3. Potentials}

The following definition of a nonlinear potential is based on the discussion from the introduction, and, as will be seen in examples, includes definitions of nonlinear potentials in other settings. 
Definition 3.1. We say that $u \in \mathcal{F}$ is a potential, if $u=U \phi$ for $\phi \in \mathcal{F}_{+}^{*}$. The function $U \phi$ is called the potential of $\phi$. The set of all potentials will be denoted by $\mathcal{P}$.

Note that since $U$ is $1-1$ and onto, the representation $u=U \phi$ is unique. Importance of potentials lies in the following observation which easily follows from Theorem 2.1: For each $u \in \mathcal{F}$ there exists a potential $v \in \mathcal{P}$ such that $v \geq u$ and $\|v\| \leq\|u\|$. In various inequalities the size of a function is estimated by its norm (e.g. the strong capacitary inequality). The above observation shows that it is usually sufficient to do the estimate for potentials only. If one wanted to estimate the size of the absolute value of the function from $\mathcal{F}$, the stronger assumption $(\mathbf{D})$ would be needed.

The following theorem gives several equivalent characterizations of potentials. Equivalence of (i), (iii) and (vi) was shown in [18], of (i), (ii) and (iii) in [19, Theorem 3.1, Theorem 5.8], and (i) and (iii) in [22, Theorem 10], and in $[12$, Proposition 2.5], in respective settings. Implication (iii) $\Rightarrow$ (i) appears also in [16] as Lemma 4.1 .

THEOREM 3.2. Let $u \in \mathcal{F}$. The following are equivalent:

(i) $u$ is a potential.

(ii) $\langle J u, v\rangle \geq 0$ for every $v \in \mathcal{F}_{+}$(i.e., Ju $\in \mathcal{F}_{+}^{*}$ ).

(iii) For every $v \in \mathcal{F}$ such that $v \geq u$ it follows that $\|v\| \geq\|u\|$.

(iv) $\min \left\{Q(\psi): \psi \in \mathcal{F}_{+}^{*}\right\}=\min \left\{Q(\psi): \psi \in \mathcal{F}^{*}\right\}$.

(v) $\sup \left\{\langle\phi, u\rangle: \phi \in \mathcal{F}_{+}^{*},\|\phi\| \leq 1\right\}=\sup \left\{\langle\phi, u\rangle: \phi \in \mathcal{F}^{*},\|\phi\| \leq 1\right\}$.

(vi) $u \geq 0$ and $c(\tilde{u})=\|u\|$.

Remark. In (iv), $Q=Q_{u}$. In (vi), $c(\tilde{u})$ denotes the capacity of the quasicontinuous modification of $u$. Although we do not deal with these notions in the present paper, (vi) is included for completness.

Proof. We prove here the equivalence of (i), (ii) and (iii), and leave the rest to Section 4.

(i) $\Leftrightarrow$ (ii): This is immediate from definition and Proposition 2.2.

(iii) $\Rightarrow$ (ii): Let $\left\|u_{0}\right\|=\min \{\|v\|: v \geq u\}$. Then $u_{0} \geq u,\left\|u_{0}\right\| \leq\|u\|$, and $J u_{0} \geq 0$. By the assumption $\left\|u_{0}\right\| \geq\|u\|$, and therefore by uniqueness $u_{0}=u$. Hence $J u \geq 0$.

(i) $\Rightarrow$ (iii): Again, let $\left\|u_{0}\right\|=\min \{\|v\|: v \geq u\}$. Then $u_{0} \geq u, u_{0} \geq 0$, $\left\|u_{0}\right\| \leq\|u\|$, and $J u_{0} \geq 0$. Since $u$ is a potential, $J u \geq 0$. Therefore, $0 \leq\left\langle J u, u_{0}-u\right\rangle$, i.e., $\langle J u, u\rangle \leq\left\langle J u, u_{0}\right\rangle$. By $(2.1),\|J u\|\|u\| \leq\|J u\|\left\|u_{0}\right\|$, 
implying that $\|u\| \leq\left\|u_{0}\right\|$. By uniqueness, $u_{0}=u$, and therefore $\|u\| \leq\|v\|$ for every $v \in \mathcal{F}$ satisfying $v \geq u$.

The next corollary should be compared with Theorem 3.1 in [9].

Corollary 3.3. Let $u \in \mathcal{F}$. There exist $u_{1}$ and $u_{2}$ in $\mathcal{F}_{+}$such that $u=u_{1}-u_{2}$ and $u_{1}$ is a potential. Moreover, $\left\langle J u_{1}, u_{2}\right\rangle=0$.

Proof. Let $\left\|u_{1}\right\|=\inf \{\|v\|: v \in \mathcal{F}, v \geq u\}$. Then $u_{1}$ is a potential, $u_{1} \geq u$, and $\left\|u_{1}\right\| \leq\|u\|$. Let $u_{2}:=u_{1}-u$. Then $u=u_{1}-u_{2}$ and $u_{2} \geq 0$. Next, let $w \in \mathcal{F}_{+}$. Then for every $t \geq 0, u_{1}+t\left(w-u_{2}\right)=(1-t) u_{1}+t(w+u) \geq$ $(1-t) u+t(w+u)=u+t w \geq u$. Therefore, $\left\langle J u_{1}, w-u_{2}\right\rangle \geq 0$ for every $w \geq 0$. By taking $w=0$ and $w=2 u_{2}$, it follows that $\left\langle J u_{1}, u_{2}\right\rangle=0$.

An analog of Cartan's result on completness of potentials is valid, and has an easy proof.

THEOREM 3.4. The set $\mathcal{P}$ of all potentials is complete.

Proof. It suffices to show that $\mathcal{P}$ is closed in $\mathcal{F}$. Let $\left\{u_{n}: n \in \mathbb{N}\right\}$ be a sequence of potentials such that $u_{n} \rightarrow u$ in $\mathcal{F}$. Since the duality mapping $J$ is norm to weak-* continuous (e.g. [8, Theorem I.4.12]), it follows that $J u_{n} \rightarrow J u$ weak-* in $\mathcal{F}^{*}$. Let $f \in \mathcal{F}, f \geq 0$. Then $\langle J u, f\rangle=\lim _{n}\left\langle J u_{n}, f\right\rangle \geq$ 0 , because $J u_{n} \in \mathcal{F}_{+}^{*}$. It follows that $J u \in \mathcal{F}_{+}^{*}$, and therefore $u=U(J u) \in$ $\mathcal{P}$.

In the next proposition we show, under the additional assumption of uniform convexity of $\mathcal{F}$, that the family of potentials is closed under monotone limits. Moreover, proofs of both parts show the usefulness of characterization of potentials by property (iii) in Theorem 3.2.

Proposition 3.5. Assume that $\mathcal{F}$ is uniformly convex.

(a) Let $0 \leq u_{1} \leq u_{2} \leq \cdots$ be an increasing sequence of potentials such that $\sup \left\{\left\|u_{n}\right\|: n \in \mathbb{N}\right\}<\infty$. Let $u=\lim _{n} u_{n} \mathcal{N}$-a.e. Then $u \in \mathcal{F}$ and $u$ is also a potential.

(b) Let $u_{1} \geq u_{2} \geq \cdots$ be a decreasing sequence of potentials, and let $u=$ $\lim _{n} u_{n} \mathcal{N}$-a.e. Then $u \in \mathcal{F}$ and $u$ is also a potential. 
Proof. (a) Let $\rho:=\sup \left\{\left\|u_{n}\right\|: n \in \mathbb{N}\right\}<\infty$. Since every $u_{n}$ is a potential, the sequence $\left\{\left\|u_{n}\right\|: n \in \mathbb{N}\right\}$ is increasing, and therefore, $\rho=$ $\lim _{n}\left\|u_{n}\right\|$. Let us consider the sequence $\left\{u_{n} / \rho: n \in \mathbb{N}\right\}$. Clearly,

$$
\lim _{n}\left\|u_{n} / \rho\right\|=1
$$

Further, for $m<n$ it holds that $(1 / 2)\left(u_{m}+u_{n}\right) \geq u_{m}$. Since $u_{m}$ is a potential, $\left\|(1 / 2)\left(u_{m}+u_{n}\right)\right\| \geq\left\|u_{m}\right\|$. It follows that

$$
\liminf _{m, n \rightarrow \infty}\left\|\frac{1}{2}\left(\frac{u_{m}}{\rho}+\frac{u_{n}}{\rho}\right)\right\| \geq \liminf _{m}\left\|\frac{u_{m}}{\rho}\right\|=1 .
$$

From (3.1) and (3.2) it follows that the sequence $\left\{u_{n} / \rho: n \in \mathbb{N}\right\}$ converges (strongly) in $\mathcal{F}$ (e.g., [1, Corollary 1.3.3]). Hence, $\left\{u_{n}: n \in \mathbb{N}\right\}$ also converges in $\mathcal{F}$, and by hypothesis $(\mathbf{C})$ it easily follows that the limit function is equal to $u$. Thus $u \in \mathcal{F}$, and clearly, $\|u\|=\rho$. Suppose now that $v \in \mathcal{F}$ such that $v \geq u$. Then $v \geq u_{n}$, hence $\|v\| \geq\left\|u_{n}\right\|$, for every $n \in \mathbb{N}$. Therefore, $\|v\| \geq\|u\|$, and by Theorem 3.2, $u$ is a potential.

(b) By boundedness of $\left\{\left\|u_{n}\right\|: n \in \mathbb{N}\right\}$ and reflexivity of $\mathcal{F}$, we may assume, without loss of generality, that $u_{n} \rightarrow v$ weakly for some $v \in \mathcal{F}$. By a corollary of Mazur's lemma, there is a sequence of $\left\{v_{n}: n \in \mathbb{N}\right\}$ of convex combinations of $\left\{u_{n}: n \in \mathbb{N}\right\}$ such that $v_{n} \rightarrow v$ in $\mathcal{F}$. More precisely, for each $n \in \mathbb{N}, v_{n}=\sum_{j=1}^{k_{n}} \alpha_{n j} u_{j}, \alpha_{n j} \geq 0, j=1, \ldots, k_{n}$, $\sum_{j=1}^{k_{n}} \alpha_{n j}=1$. Note that $v_{n} \geq \sum_{j=1}^{k_{n}} \alpha_{n j} u_{k_{n}}=u_{k_{n}}$, for every $n \in \mathbb{N}$. Since $u_{k_{n}}$ is a potential, it holds that $\left\|v_{n}\right\| \geq\left\|u_{k_{n}}\right\|$, for every $n \in \mathbb{N}$. Therefore, $\lim \sup _{n}\left\|u_{k_{n}}\right\| \leq \lim \left\|v_{n}\right\|=\|v\|$. On the other hand, $u_{k_{n}} \rightarrow v$ weakly, and so $\liminf _{n}\left\|u_{k_{n}}\right\| \geq\|v\|$ by the weak lower semicontinuity of the norm. These two inequalities imply that $\lim _{n}\left\|u_{k_{n}}\right\|=\|v\|$. Since $\mathcal{F}$ was assumed to be uniformly convex, it follows that $u_{k_{n}} \rightarrow v$ in $\mathcal{F}$. By Theorem 3.4, $v \in \mathcal{P}$. Moreover, by the hypothesis $(\mathbf{C}), v=u \mathcal{N}$-a.e. Thus $u \in \mathcal{P}$.

Remark. Suppose that $0 \leq u_{1} \leq u_{2} \leq \cdots$ is an increasing sequence of potentials converging $\mathcal{N}$-a.e. to $u$. If we assume that $u \in \mathcal{F}$, then $u \geq$ $u_{n}$ and the fact that $u_{n}$ is a potential, imply that $\left\|u_{n}\right\| \leq\|u\|$. Hence, $\sup _{n}\left\{\left\|u_{n}\right\|, n \in \mathbb{N}\right\}<\infty$, and therefore, $u$ is a potential.

\section{§4. Reduced functions}

Let $B \subset X$. For $u, v \in \mathcal{F}$ we say that $v \geq u$ on $B$ (as equivalence classes) if $v \geq u \mathcal{N}$-a.e. on $B$ (as functions). This means that there is 
$N \in \mathcal{N}$ such that $v(x) \geq u(x)$ for every $x \in B \backslash N$. Let

$$
\mathcal{K}_{u, B}:=\{v \in \mathcal{F}: v \geq u \text { on } B\} .
$$

Then $\mathcal{K}_{u, B}$ is convex and closed in $\mathcal{F}$. Moreover, if $v \in \mathcal{K}_{u, B}$ and $w \in \mathcal{F}_{+}$, then $v+w \in \mathcal{K}_{u, B}$. Thus the following theorem is a direct consequence of Theorem 2.1

TheOREM 4.1. Let $u \in \mathcal{F}$. There exists a unique $u_{B} \in \mathcal{K}_{u, B}$ such that

$$
\left\|u_{B}\right\|=\min \{\|v\|: v \geq u \text { on } B\}
$$

Moreover, $u_{B}$ is a potential and there is a unique $\phi_{B} \in \mathcal{F}_{+}^{*}$ such that $u_{B}=$ $U \phi_{B}$.

Definition 4.2. The potential $u_{B}=U \phi_{B}$ is called the reduced function of $u$ onto $B$.

Remark. We note that $\left(u_{B}\right)_{B}=u_{B}$. Indeed, since $u_{B} \geq u$ on $B$, it follows that

$$
\begin{aligned}
\left\|\left(u_{B}\right)_{B}\right\| & =\inf \left\{\|v\|: v \geq u_{B} \text { on } B\right\} \\
& \geq \inf \{\|v\|: v \geq u \text { on } B\}=\left\|u_{B}\right\| .
\end{aligned}
$$

But clearly, $\left\|\left(u_{B}\right)_{B}\right\| \leq\left\|u_{B}\right\|$. Therefore, $\left(u_{B}\right)_{B}=u_{B}$.

Now we want to show that the functional $\phi_{B}$ representing the reduced function $u_{B}$ is "supported" by $B$. To make this precise, let

$$
\mathcal{F}_{X \backslash B,+}:=\{v \in \mathcal{F}: v \geq 0 \text { on } B\} .
$$

and let $\mathcal{F}_{X \backslash B}:=\left(\mathcal{F}_{X \backslash B,+}\right) \cap\left(-\mathcal{F}_{X \backslash B,+}\right)$. Note that both $\mathcal{F}_{X \backslash B,+}$ and $\mathcal{F}_{X \backslash B}$ are closed in $\mathcal{F}$. Indeed, if $\left(v_{n}, n \in \mathbb{N}\right) \subset \mathcal{F}_{X \backslash B,+}$ and $v_{n} \rightarrow v$ in $\mathcal{F}$, then there is a subsequence $\left(v_{n_{k}}, k \in \mathbb{N}\right)$ such that $v_{n_{k}} \rightarrow v \mathcal{N}$-a.e. Therefore, $v \geq 0 \mathcal{N}$-a.e. on $B$, i.e., $v \in \mathcal{F}_{X \backslash B,+}$.

The object dual to $\mathcal{F}_{X \backslash B,+}$ is defined by

$$
\mathcal{F}_{B,+}^{*}:=\left\{\phi \in \mathcal{F}^{*}:\langle\phi, u\rangle \geq 0 \text { for every } u \in \mathcal{F}_{X \backslash B,+}\right\} .
$$

Note that $\mathcal{F}_{B,+}^{*} \subset \mathcal{F}_{+}^{*}$. Moreover, if $\mathcal{F}_{B}^{*}:=\left\{\phi \in \mathcal{F}^{*}:\langle\phi, u\rangle=0\right.$ for every $\left.u \in \mathcal{F}_{X \backslash B}\right\}$, then also $\mathcal{F}_{B,+}^{*} \subset \mathcal{F}_{B}^{*}$. It is reasonable to regard the elements of $\mathcal{F}_{B,+}^{*}$ as positive functionals "supported" by $B$. 
LEMmA 4.3. Let $u \in \mathcal{F}$ satisfy $\langle\phi, u\rangle \geq 0$ for every $\phi \in \mathcal{F}_{B,+}^{*}$. Then $u \in \mathcal{F}_{X \backslash B,+}$.

Proof. Suppose that $u \notin \mathcal{F}_{X \backslash B,+}$. Since $\mathcal{F}_{X \backslash B,+}$ is closed and convex, by the Hahn-Banach separation theorem there exists $\phi_{0} \in \mathcal{F}^{*}$ such that

$$
\left\langle\phi_{0}, u\right\rangle<\inf \left\{\left\langle\phi_{0}, v\right\rangle: v \in \mathcal{F}_{X \backslash B,+}\right\} .
$$

Let $v \in \mathcal{F}_{X \backslash B,+}$. Then $n v \in \mathcal{F}_{X \backslash B,+}$ for every $n \in \mathbb{N}$, and hence

$$
\left\langle\phi_{0}, u\right\rangle\left\langle\left\langle\phi_{0}, n v\right\rangle=n\left\langle\phi_{0}, v\right\rangle, \quad \text { for every } n \in \mathbb{N} .\right.
$$

This is possible only if $\left\langle\phi_{0}, v\right\rangle \geq 0$. Since $v \in \mathcal{F}_{X \backslash B,+}$ was arbitrary, it follows that $\phi_{0} \in \mathcal{F}_{B,+}^{*}$. On the other hand, $\left\langle\phi_{0}, u\right\rangle<\inf \left\{\left\langle\phi_{0}, v\right\rangle: v \in\right.$ $\left.\mathcal{F}_{X \backslash B,+}\right\}=0$, contradicting the assumption on $u$.

Corollary 4.4. (i) If $\langle\phi, u\rangle \geq 0$ for every $\phi \in \mathcal{F}_{+}^{*}$, then $u \in \mathcal{F}_{+}$. (ii) $\mathcal{F}_{B,+}^{*}=\{0\}$ if and only if $B \in \mathcal{N}$.

Let $u \in \mathcal{F}$ be fixed, and recall that $Q=Q_{u}: \mathcal{F}^{*} \rightarrow \mathbb{R}$ was defined by $(2.5)$ as $Q(\phi)=\Gamma^{*}(\|\phi\|)-\langle\phi, u\rangle$.

Lemma 4.5. Let $\phi \in \mathcal{F}^{*}$ and let $\|\phi\|=1$. Then $t \mapsto Q(t \phi), t \in \mathbb{R}$, attains its minimum for $|t| \leq \gamma(\|u\|)$.

Proof. Since $\|\phi\|=1$, it follows that $Q(t \phi)=\Gamma^{*}(|t|)-t\langle\phi, u\rangle$. Further,

$$
\frac{d}{d t} \Gamma^{*}(t \phi)=(\operatorname{sign} t) \gamma^{*}(t)-\langle\phi, u\rangle
$$

It easily follows that the minimum is attained for $|t| \leq \gamma(|\langle\phi, u\rangle|) \leq$ $\gamma(\|\phi\|\|u\|)=\gamma(\|u\|)$.

Let $\mathcal{G}^{*} \subset \mathcal{F}^{*}$ be any closed subset of functionals having a nonempty intersection with the closed ball $\left\{\phi \in \mathcal{F}^{*}:\|\phi\| \leq \gamma(\|u\|)\right\}$. Lemma 4.5 shows that

$$
\inf \left\{Q(\phi): \phi \in \mathcal{G}^{*}\right\}=\inf \left\{Q(\phi): \phi \in \mathcal{G}^{*},\|\phi\| \leq \gamma(\|u\|)\right\}
$$

Moreover, by the Banach-Alaoglu theorem, $\mathcal{G}^{*} \cap\{\phi:\|\phi\| \leq \gamma(\|u\|)\}$ is weak-* compact. Therefore, the infimum of the right hand side, and hence of the left hand side, is attained. 
Lemma 4.6. Let $\mathcal{G}^{*}$ be a closed and convex subset of $\mathcal{F}^{*}$ having a nonempty intersection with the closed ball of radius $\gamma(\|u\|)$. Then there exists a unique $\psi \in \mathcal{G}^{*}$ such that

$$
Q(\psi)=\min \left\{Q(\phi): \phi \in \mathcal{G}^{*}\right\} .
$$

Proof. Existence of such a $\psi$ was shown before the lemma. Suppose that there are two functionals, $\psi_{1}, \psi_{2} \in \mathcal{G}^{*}$ minimizing the right hand side in (4.2). By convexity of $\mathcal{G}^{*}, \psi:=\left(\psi_{1}+\psi_{2}\right) / 2 \in \mathcal{G}^{*}$. By strict convexity of $\mathcal{F}^{*}$ and convexity of $\Gamma^{*}$ the following sequence of inequalities is valid:

$$
\begin{aligned}
Q(\psi) & =\Gamma^{*}(\|\psi\|)-\langle\psi, u\rangle \\
& <\Gamma^{*}\left(\frac{1}{2}\left\|\psi_{1}\right\|+\frac{1}{2}\left\|\psi_{2}\right\|\right)-\frac{1}{2}\left\langle\psi_{1}, u\right\rangle-\frac{1}{2}\left\langle\psi_{2}, u\right\rangle \\
& \leq \frac{1}{2} \Gamma^{*}\left(\left\|\psi_{1}\right\|\right)+\frac{1}{2} \Gamma^{*}\left(\left\|\psi_{2}\right\|\right)-\frac{1}{2}\left\langle\psi_{1}, u\right\rangle-\frac{1}{2}\left\langle\psi_{2}, u\right\rangle \\
& =\frac{1}{2} Q\left(\psi_{1}\right)+\frac{1}{2} Q\left(\psi_{2}\right)=Q\left(\psi_{1}\right)
\end{aligned}
$$

But this contradicts the fact that $\psi_{1}$ minimizes the right hand side in (4.2).

Let $B \subset X$, let $u \in \mathcal{F}$, and let $u_{B}=U \phi_{B}$ be the reduced function of $u$ onto $B$. The Gauss-Frostman approach to reduced functions consists of solving the following minimizing problem:

$$
\min \left\{Q(\psi): \psi \in \mathcal{F}_{B,+}^{*}\right\} .
$$

By taking $\mathcal{G}^{*}=\mathcal{F}_{B,+}^{*}$ in Lemma 4.6, we conclude that there exists a unique $\psi_{B} \in \mathcal{F}_{B,+}^{*}$ such that

$$
Q\left(\psi_{B}\right)=\min \left\{Q(\psi): \psi \in \mathcal{F}_{B,+}^{*}\right\} .
$$

THEOREM 4.7. The potential of $\psi_{B}$ is equal to the reduced function of $u$ onto $B: U \psi_{B}=u_{B}$. In particular $\phi_{B}=\psi_{B}$.

Proof. Recall equation (2.3) according to which for every $\psi \in \mathcal{F}^{*}$

$$
\frac{d}{d t} \Gamma^{*}\left(\left\|\psi_{B}+t \psi\right\|\right)_{\mid t=0}=\left\langle\psi, U \psi_{B}\right\rangle
$$


Let $t \geq 0$ and $\psi \in \mathcal{F}_{B,+}^{*}$. Then $\psi_{B}+t \psi \in \mathcal{F}_{B,+}^{*}$ and therefore $Q\left(\psi_{B}+t \psi\right) \geq$ $Q\left(\psi_{B}\right)$ for every $t \geq 0$. Therefore

$$
\frac{d}{d t} Q\left(\psi_{B}+t \psi\right)_{\mid t=0} \geq 0
$$

By using (4.5), it follows that

$$
\frac{d}{d t} Q\left(\psi_{B}+t \psi\right)_{\mid t=0}=\left\langle\psi, U \psi_{B}\right\rangle-\langle\psi, u\rangle
$$

Therefore,

$$
\left\langle\psi, U \psi_{B}-u\right\rangle \geq 0 \text { for every } \psi \in \mathcal{F}_{B,+}^{*} .
$$

By Lemma 4.3 it follows that $U \psi_{B}-u \in \mathcal{F}_{X \backslash B,+}$, i.e., $U \psi_{B} \geq u$ on $B$.

Further, notice that $\psi_{B}+t \psi_{B} \in \mathcal{F}_{B,+}^{*}$ for every $t \in(-1, \infty)$. This is clear for $t \geq 0$, while for $t \in(-1,0), \psi_{B}+t \psi_{B}=(1+t) \psi_{B} \in \mathcal{F}_{B,+}^{*}$ since $1+t>0$. Therefore, $Q\left(\psi_{B}+t \psi_{B}\right) \geq Q\left(\psi_{B}\right)$ for every $t>-1$. This implies that

$$
0=\frac{d}{d t} Q\left(\psi_{B}+t \psi_{B}\right)_{\mid t=0}=\left\langle\psi_{B}, U \psi_{B}-u\right\rangle .
$$

Further, since $u_{B} \geq u$ on $B$, it follows that $\left\langle\psi_{B}, u_{B}-u\right\rangle \geq 0$. Hence, $\left\langle\psi_{B}, u_{B}-U \psi_{B}\right\rangle=\left\langle\psi_{B}, u_{B}-u\right\rangle+\left\langle\psi_{B}, u-U \psi_{B}\right\rangle \geq 0$. Therefore, $\left\|\psi_{B}\right\|\left\|U \psi_{B}\right\|=\left\langle\psi_{B}, U \psi_{B}\right\rangle \leq\left\langle\psi_{B}, u_{B}\right\rangle \leq\left\|\psi_{B}\right\|\left\|u_{B}\right\|$. If $\left\|\psi_{B}\right\| \neq 0$, it follows that $\left\|U \psi_{B}\right\| \leq\left\|u_{B}\right\|$. But $U \psi_{B} \geq u$ on $B$. Hence by uniquness of the reduced function, it follows that $U \psi_{B}=u_{B}$. The case when $\left\|\psi_{B}\right\|=0$ may happen only when $u \in-\left(\mathcal{F}_{X \backslash B,+}\right)$, when clearly $u_{B}=0$ and the zero functional minimizes $Q$.

By taking $B \subset X$ such that $X \backslash B \in \mathcal{N}$ (or simply $B=X$ ), we get that $\mathcal{F}_{X \backslash B,+}=\mathcal{F}_{+}$and $\mathcal{F}_{B,+}^{*}=\mathcal{F}_{+}^{*}$. Let $u \in \mathcal{F}$, and let $\left\|u_{0}\right\|=\left\|U \phi_{0}\right\|=$ $\min \{v \in \mathcal{F}: v \geq u\}$. If

$$
Q\left(\psi_{0}\right)=\min \left\{Q(\psi): \psi \in \mathcal{F}_{+}^{*}\right\},
$$

then by Theorem 4.7, $u_{0}=U \psi_{0}$. We may now prove the equivalence of (i) and (iv) from Theorem 3.2. Let $\widehat{\psi}_{0} \in \mathcal{F}^{*}$ minimize the right hand side in (iv). Then $Q\left(\widehat{\psi}_{0}+t \psi\right) \geq Q\left(\widehat{\psi}_{0}\right)$ for every $\psi \in \mathcal{F}^{*}$ and every $t \in \mathbb{R}$. Therefore,

$$
0=\frac{d}{d t} Q\left(\widehat{\psi}_{0}+t \psi\right)_{\mid t=0}=\left\langle\psi, U \widehat{\psi}_{0}-u\right\rangle \quad \text { for every } \psi \in \mathcal{F}^{*} .
$$


This implies that $u=U \widehat{\psi}_{0}$.

(i) $\Rightarrow\left(\right.$ iv): If $u$ is a potential, then $\widehat{\psi}_{0}=J u \geq 0$, hence $\widehat{\psi}_{0}$ minimizes $Q$ over nonnegative functionals as well.

(iv) $\Rightarrow$ (i): If $Q\left(\widehat{\psi}_{0}\right)=\min \left\{Q(\psi): \psi \in \mathcal{F}_{+}^{*}\right\}$, then $\widehat{\psi}_{0} \in \mathcal{F}_{+}^{*}$, and hence $u=U \widehat{\psi}_{0}$ is a potential.

Here is a simple corollary of the fact that $\phi_{B} \in \mathcal{F}_{B,+}^{*}$.

Corollary 4.8. Let $u \in \mathcal{F}, B \subset X$, and let $u_{B}=U \phi_{B}$ be the reduced function of $u$ onto $B$. Let $v \in \mathcal{F}$ be such that $v \geq u$ on $B$. Then $\left\langle\phi_{B}, u_{B}\right\rangle \leq$ $\left\langle\phi_{B}, v\right\rangle$.

Proof. Note that for $t \in[0,1]$ it holds that $u_{B}+t\left(v-u_{B}\right)=(1-$ $t) u_{B}+t v \geq u$ on $B$. Therefore, $\left\|u_{B}+t\left(v-u_{B}\right)\right\| \geq\left\|u_{B}\right\|$ for every $t \in[0,1]$. Hence,

$$
0 \leq\left\langle J u_{B}, v-u_{B}\right\rangle=\left\langle\phi_{B}, v-u_{B}\right\rangle
$$

Alternatively, since $\left\langle\phi_{B}, u-u_{B}\right\rangle=0$, it follows immediately that $\left\langle\phi_{B}, v-u_{B}\right\rangle=\left\langle\phi_{B}, v-u\right\rangle+\left\langle\phi_{B}, u-u_{B}\right\rangle \geq 0$.

Let again $B \subset X$, and let $u \in \mathcal{F}$. We consider the following dual problem:

$$
\sup \left\{\langle\phi, u\rangle: \phi \in \mathcal{F}_{B,+}^{*},\|\phi\| \leq 1\right\}
$$

Note that $\phi \mapsto\langle\phi, u\rangle$ is weak-* continuous. Since the set $\mathcal{F}_{B,+}^{*} \cap\{\phi:\|\phi\| \leq$ $1\}$ is weak-* compact, the mapping $\phi \mapsto\langle\phi, u\rangle$ attains its maximum on that set. Let $\chi_{B} \in \mathcal{F}_{B,+}^{*},\left\|\chi_{B}\right\| \leq 1$ be the maximizing functional:

$$
\left\langle\chi_{B}, u\right\rangle=\max \left\{\langle\phi, u\rangle: \phi \in \mathcal{F}_{B,+}^{*},\|\phi\| \leq 1\right\}
$$

Suppose that $u \leq 0$ on $B$. For every $\phi \in \mathcal{F}_{B,+}^{*}$ it holds that $\langle\phi, u\rangle \leq$ 0 . Therefore, $\chi_{B}=0$. Moreover, note that for such $u$ it holds that $u_{B}=U \phi_{B}=0$ and therefore $\phi_{B}=0$. From now on we assume that $u \notin-\mathcal{F}_{X \backslash B,+}$.

Lemma 4.9. Suppose that $u \notin-\mathcal{F}_{X \backslash B,+}$. There exists a unique $\chi_{B}$ such that (4.9) holds true. Moreover, $\left\|\chi_{B}\right\|=1$. 
Proof. The existence of $\chi_{B}$ with $\left\|\chi_{B}\right\| \leq 1$ was shown. Note that there exists $\phi \in \mathcal{F}_{B,+}^{*}$ such that $\langle\phi, u\rangle>0$. Indeed, if $\langle\phi, u\rangle \leq 0$ for every $\phi \in \mathcal{F}_{B,+}^{*}$, then it follows by Lemma 4.3 that $u \leq 0$ on $B$, contrary to the assumption.

This implies that $\left\langle\chi_{B}, u\right\rangle>0$. If $\left\|\chi_{B}\right\|<1$, then

$$
\left\langle\frac{\chi_{B}}{\left\|\chi_{B}\right\|}, u\right\rangle>\left\langle\chi_{B}, u\right\rangle \text {. }
$$

Therefore, $\left\|\chi_{B}\right\|=1$.

Suppose that $\chi_{1}$ and $\chi_{2}$ are two functionals in $\mathcal{F}_{B,+}^{*}$ maximizing the right hand side in (4.8) both having norm equal to 1 . Define $\chi:=\left(\chi_{1}+\right.$ $\left.\chi_{2}\right) / 2$. Then $\chi \in \mathcal{F}_{B,+}^{*},\|\chi\|<1$ (by the strict convexity of $\mathcal{F}^{*}$ ), and clearly

$$
\langle\chi, u\rangle=\max \left\{\langle\phi, u\rangle: \phi \in \mathcal{F}_{B,+}^{*},\|\phi\| \leq 1\right\} .
$$

But every maximizing functional is of norm 1, contradicting the fact that $\|\chi\|<1$. Therefore, $\chi_{B}$ is unique.

Theorem 4.10. Let $B \subset X$, let $u \in \mathcal{F}, u \notin-\mathcal{F}_{X \backslash B,+}$, and let $u_{B}=$ $U \phi_{B}$ be the reduced function of $u$ onto $B$. Let $\chi_{B}$ satisfy (4.9). Then

$$
\chi_{B}=\frac{\phi_{B}}{\left\|\phi_{B}\right\|} .
$$

Proof. Recall from Theorem 4.7 that $\phi_{B}$ minimizes $Q$ over all functionals in $\mathcal{F}_{B,+}^{*}$. Therefore

$$
\Gamma^{*}\left(\left\|\phi_{B}\right\|\right)-\left\langle\phi_{B}, u\right\rangle=Q\left(\phi_{B}\right) \leq Q\left(\left\|\phi_{B}\right\| \chi_{B}\right)=\Gamma^{*}\left(\left\|\phi_{B}\right\|\right)-\left\|\phi_{B}\right\|\left\langle\chi_{B}, u\right\rangle .
$$

It follows that

$$
\left\langle\chi_{B}, u\right\rangle \leq\left\langle\frac{\phi_{B}}{\left\|\phi_{B}\right\|}, u\right\rangle .
$$

By uniqueness of $\chi_{B}$ it follows that $\chi_{B}=\phi_{B} /\left\|\phi_{B}\right\|$.

Having identified the functional maximizing (4.8), it is now easy to compute the value of the maximum. Indeed,

$$
\left\langle\chi_{B}, u\right\rangle=\left\langle\frac{\phi_{B}}{\left\|\phi_{B}\right\|}, u\right\rangle=\frac{1}{\left\|\phi_{B}\right\|}\left\langle\phi_{B}, U \phi_{B}\right\rangle=\left\|U \phi_{B}\right\|
$$

because $\left\langle\phi_{B}, U \phi_{B}\right\rangle=\left\|\phi_{B}\right\|\left\|U \phi_{B}\right\|$. Thus, the following corollary holds. 
Corollary 4.11. For $B \subset X$ and $u \in \mathcal{F}$ we have

$$
\begin{aligned}
\inf \{\|v\|: v \in \mathcal{F}, v \geq u \text { on } B\} & =\left\|u_{B}\right\| \\
& =\sup \left\{\langle\phi, u\rangle: \phi \in \mathcal{F}_{B,+}^{*},\|\phi\| \leq 1\right\}
\end{aligned}
$$

We can now prove the equivalence of (i) and (v) from Theorem 3.2. Note that $\sup \left\{\langle\phi, u\rangle: \phi \in \mathcal{F}^{*},\|\phi\| \leq 1\right\}=\|u\|$, and the unique functional for which the maximum is attained is $J u /\|J u\|$. If $u$ is a potential, then $J u \in \mathcal{F}_{+}^{*}$, and therefore (v) holds. If (v) holds, then it follows that $J u \geq 0$, hence $u$ is a potential.

The following proposition compares the reduced functions onto two different sets.

Proposition 4.12. Let $B \subset C \subset X$, let $u \in \mathcal{F}$, and let $u_{B}=U \phi_{B}$ and $u_{C}$, be the reduced functions of $u$ onto $B$ and $C$, respectively. Then

$$
\left\langle\phi_{B}, u_{C}-u_{B}\right\rangle \geq 0 .
$$

Proof. Let $u_{C}=U \phi_{C}$, and let $\chi_{B}=\phi_{B} /\left\|\phi_{B}\right\|$ and $\chi_{C}=\phi_{C} /\left\|\phi_{C}\right\|$. Then

$$
\begin{aligned}
& \left\langle\chi_{B}, u\right\rangle=\sup \left\{\langle\phi, u\rangle: \phi \in \mathcal{F}_{B,+}^{*},\|\phi\| \leq 1\right\} \\
& \left\langle\chi_{C}, u\right\rangle=\sup \left\{\langle\phi, u\rangle: \phi \in \mathcal{F}_{C,+}^{*},\|\phi\| \leq 1\right\}
\end{aligned}
$$

Since $\mathcal{F}_{X \backslash B,+} \supset \mathcal{F}_{X \backslash C,+}$, it follows that $\mathcal{F}_{B,+}^{*} \subset \mathcal{F}_{C,+}^{*}$, and therefore $\left\langle\chi_{B}, u\right\rangle \leq\left\langle\chi_{C}, u\right\rangle$. Note that $\left\langle\chi_{B}, u\right\rangle=\left\langle\chi_{B}, u_{B}\right\rangle$ (see (4.7)). Further, since $u_{C} \geq u$ on $C \supset B$, it follows that $\left\langle\chi_{B}, u_{C}-u\right\rangle \geq 0$, i.e., $\left\langle\chi_{B}, u\right\rangle \leq\left\langle\chi_{B}, u_{C}\right\rangle$. Therefore, $\left\langle\chi_{B}, u_{B}\right\rangle=\left\langle\chi_{B}, u\right\rangle \leq\left\langle\chi_{B}, u_{C}\right\rangle$, i.e., $\left\langle\phi_{B}, u_{C}-u_{B}\right\rangle \geq 0$.

We will now show another convergence result. For the proof we need the result due to S. Kakutani saying that every uniformly convex Banach space has the following Banach-Saks property: Every bounded sequence has a subsequence whose arithmetic means converge in norm (e.g. [10, p. 78]).

Proposition 4.13. Assume that both $\mathcal{F}$ and $\mathcal{F}^{*}$ are uniformly convex. Let $B \subset X$, let $u \in \mathcal{F}, u \geq 0$, and let $\left\{u_{n} ; n \in \mathbb{N}\right\} \subset \mathcal{F}$ be a sequence such that $u_{1} \geq u_{2} \geq \cdots$ on $B$ and $\lim _{n} u_{n}=u$ on $B$. Assume that $\sup \left\{\left\|u_{n}\right\|\right.$ : $n \in \mathbb{N}\}<\infty$. Let $\left(u_{n}\right)_{B}$ be the reduced function of $u_{n}$ onto $B$. Then $\left(u_{n}\right)_{B} \rightarrow u_{B}$ in $\mathcal{F}$. 
Proof. First note that since $u_{n+1} \leq u_{n}$ and $\left(u_{n}\right)_{B} \geq u_{n}$ on $B$, it follows that $\left(u_{n}\right)_{B} \geq u_{n+1}$ on $B$. Therefore, $\left\|\left(u_{n}\right)_{B}\right\| \geq\left\|\left(u_{n+1}\right)_{B}\right\|$. Also, $\sup \left\{\left\|\left(u_{n}\right)_{B}\right\|: n \in \mathbb{N}\right\}<\infty$. By passing to a subsequence, we may assume that there are $u^{\prime}, v \in \mathcal{F}$ such that $u_{n} \rightarrow u^{\prime}$ and $\left(u_{n}\right)_{B} \rightarrow v$ weakly, and arithmetic means $w_{n}$ of $\left\{\left(u_{n}\right)_{B}: n \in \mathbb{N}\right\}$ converge to $v$ strongly. Clearly, $w_{n} \geq u_{n}$ on $B$, and hence, $\left\|w_{n}\right\| \geq\left\|\left(u_{n}\right)_{B}\right\|$. Similarly as in the proof of Proposition 3.5 (ii), it follows that $\left(u_{n}\right)_{B} \rightarrow v$ strongly in $\mathcal{F}$, and $v$ is a potential. Moreover, by $(\mathbf{C}), v \geq u$ on $B$, and hence, $\|v\| \geq\left\|u_{B}\right\|$. Let $\left(u_{n}\right)_{B}=U \phi_{n}$ and $v=U \phi$. By uniform convexity of $\mathcal{F}^{*}, J$ is norm to norm continuous (e.g. [8, Theorem II.2.16]). Therefore, $\phi_{n} \rightarrow \phi$ in $\mathcal{F}^{*}$. Since $\phi_{n} \in \mathcal{F}_{B,+}^{*}$, it follows that $\phi \in \mathcal{F}_{B,+}^{*}$ as well. Further, $\left\langle\phi_{n}-\phi, u_{n}\right\rangle \leq$ $\left\|\phi_{n}-\phi\right\|\left\|u_{n}\right\| \rightarrow 0$. Next, by equation (4.2),

$$
\left\langle\phi_{n},\left(u_{n}\right)_{B}\right\rangle=\left\langle\phi_{n}, u_{n}\right\rangle
$$

for every $n \in \mathbb{N}$. Since $u_{n} \rightarrow u^{\prime}$ weakly, arithmetic means converge strongly, and by $(\mathbf{C}), \mathcal{N}$-a.e. Hence, $u^{\prime}=u$ on $B$. Therefore, $\left\langle\phi, u_{n}\right\rangle \rightarrow\left\langle\phi, u^{\prime}\right\rangle=$ $\langle\phi, u\rangle$. It follows from (4.12) that

$$
\lim _{n}\left\langle\phi_{n},\left(u_{n}\right)_{B}\right\rangle=\lim _{n}\left\langle\phi_{n}, u_{n}\right\rangle=\lim _{n}\left\langle\phi, u_{n}\right\rangle+\lim _{n}\left\langle\phi_{n}-\phi, u_{n}\right\rangle=\langle\phi, u\rangle .
$$

Let $\chi \in \mathcal{F}_{B,+}^{*},\|\chi\| \leq 1$. Then $\left\langle\chi, u_{n}\right\rangle \leq\left\langle\phi_{n} /\left\|\phi_{n}\right\|, u_{n}\right\rangle$. Hence,

$$
\langle\chi, u\rangle \leq\left\langle\chi, u_{n}\right\rangle \leq\left\langle\frac{\phi_{n}}{\left\|\phi_{n}\right\|}, u_{n}\right\rangle \longrightarrow\left\langle\frac{\phi}{\|\phi\|}, u\right\rangle,
$$

provided $\phi \neq 0$. Therefore,

$$
\left\langle\frac{\phi}{\|\phi\|}, u\right\rangle=\sup \left\{\langle\chi, u\rangle: \chi \in \mathcal{F}_{B,+}^{*},\|\chi\| \leq 1\right\}=\left\|u_{B}\right\|
$$

by Corollary 4.11. Let $u_{B}=U \phi_{B}$. By Theorem 4.10, $\phi /\|\phi\|=\phi_{B} /\left\|\phi_{B}\right\|$. Since we know that $\|U \phi\|=\|v\| \geq\left\|u_{B}\right\|=\left\|U \phi_{B}\right\|$, it follows $\|\phi\| \geq\left\|\phi_{B}\right\|$, and therefore $\|\phi\|=\left\|\phi_{B}\right\|$. This proves that $v=u_{B}$. The case when $\phi=0$ is even simpler.

Since this holds for every subsequence, the claim of the proposition follows.

It is possible to consider a concept of a reduced function for a function which does not necessarily belong to $\mathcal{F}$. Indeed, let $f: X \rightarrow \mathbb{R}$ be an $\mathcal{N}$-a.e. defined function. For $B \subset X$ assume that the set $\mathcal{K}_{f, B}=\{v \in \mathcal{F}$ : 
$v \geq f$ on $B\}$ is nonempty. Then exactly as before we may conclude that there exists a unique potential $f_{B}=U \phi_{B}$ such that

$$
\left\|f_{B}\right\|=\inf \left\{\|v\| ; v \in \mathcal{K}_{f, B}\right\} .
$$

Let us show that $\phi_{B} \in \mathcal{F}_{B,+}^{*}$. Look at $\left(f_{B}\right)_{B}$ defined uniquely by

$$
\left\|\left(f_{B}\right)_{B}\right\|=\inf \left\{\|v\|: v \in \mathcal{F}, v \geq f_{B} \text { on } B\right\} .
$$

Note that $\left(f_{B}\right)_{B} \geq f_{B}$ on $B$, and hence $\left(f_{B}\right)_{B} \geq f$ on $B$. Therefore $\left\|f_{B}\right\| \geq$ $\left\|\left(f_{B}\right)_{B}\right\|$. By definition $\left\|f_{B}\right\| \geq\left\|\left(f_{B}\right)_{B}\right\|$. By uniqueness, $\left(f_{B}\right)_{B}=f_{B}=$ $U \phi_{B}$. Since $f_{B} \in \mathcal{F}$, the fact that $\phi_{B} \in \mathcal{F}_{B,+}^{*}$ follows from Theorem 4.7.

\section{$\S 5 . \quad$ Examples}

We present now several examples that satisfy the introduced hypotheses.

ExAmple A. Our first example is in fact a framework for a series of examples of nonlinear potential theories with a kernel.

Let $X$ be a nonempty set and $\mathcal{N}$ an ideal of subsets of $X$. Let $\mathcal{G}$ denote the vector space of all $\mathcal{N}$-a.e. defined functions on $X$ taking values in $[-\infty,+\infty]$ such that for every $u \in \mathcal{G},\{|u|=+\infty\} \in \mathcal{N}$. We identify functions in $\mathcal{G}$ with equivalence classes of $\mathcal{N}$-a.e. equal functions, and use the same letter $\mathcal{G}$ for the vector space of equivalence classes.

Let $Y$ be another nonempty set, and let $\left(L,\|\|_{L}\right)$ be a Banach lattice of equivalence classes of functions on $Y$. We do not specify the equivalence relation yet. Assume that $\left(L,\|\|_{L}\right)$ is a reflexive, strictly convex and smooth space. There is a natural order on $L$ : For $f, g \in L, f \leq g$ in $L$ if $f \leq g$ pointwise. We also assume that for every $f \in L$ it holds that $\||f|\|_{L} \leq$ $\|f\|_{L}$. Let $\left(L^{*},\|\|_{L^{*}}\right)$ denote the dual space of $L$. We assume that there is a linear map $V: L \rightarrow \mathcal{G}$ having the following properties:

(i) If $f \geq 0$ in $L$, then $V f \geq 0$ in $\mathcal{G}$,

(ii) If $f_{n} \rightarrow f$ in $\left(L,\|\|_{L}\right)$, then there exists a subsequence $\left(n_{k}, k \in \mathbb{N}\right)$ such that $V f_{n_{k}} \rightarrow V f \mathcal{N}$-a.e.

Let $N:=\{f \in L: V f=0\}$ be the kernel of $V$. It follows from (ii) that $N$ is closed in $L$. Let $\left(L / N,\|\|_{L / N}\right)$ be the quotient space with the quotient norm, and let $\left((L / N)^{*},\|\|_{\left.(L / N)^{*}\right)}\right.$ be its dual. Let $\pi: L \rightarrow L / N$ be the natural map with the adjoint $\pi^{*}$. Recall that $\|\pi(f)\|_{L / N}=\inf \left\{\|f+g\|_{L}\right.$ : $g \in N\}$. It follows from reflexivity, strict convexity and smoothness of $L$, that $\|\pi(f)\|_{L / N}=\|f\|_{L}$ for a unique $f \in L$. Moreover, if $J: L \rightarrow L^{*}$ 
is the duality mapping, then $f$ is characterized by the following property: $L^{*}\langle J f, g\rangle_{L}=0$ for every $g \in N$. By using this fact (among some others), one easily shows that $\left(L / N,\|\|_{L / N}\right)$ is reflexive, strictly convex and smooth. Moreover, if $I: L^{*} \rightarrow L$ is the duality mapping of weight $\gamma^{*}$, then the duality mapping $\widetilde{I}:(L / N)^{*} \rightarrow L / N$ is of the form

$$
\widetilde{I}=\pi \circ I \circ \pi^{*}
$$

We define the vector space $\mathcal{F}$ as the image of $L$ by the map $V: \mathcal{F}:=$ $V(L)$. Let $\widetilde{V}: L / N \rightarrow \mathcal{G}$ be defined by $\widetilde{V} \circ \pi=V$. Then also $\mathcal{F}=\widetilde{V}(L / N)$. For $u=V f=\widetilde{V}(\pi(f))$, let $\|u\|:=\|\pi(f)\|_{L / N}$. Note that one can choose a unique $f \in L$ such that $u=V f$ and $\|u\|=\|f\|_{L}$. We will say that such $f$ minimizes the quotient norm. Since $\widetilde{V}: L / N \rightarrow \mathcal{F}$ is an isometric isomorphism, the Banach space $(\mathcal{F},\|\|)$ is reflexive, strictly convex and smooth. Hence, hypotheses $(\mathbf{F})$ are satisfied. Hypothesis $(\mathbf{C})$ follows easily from property (ii) of $V$. Let us check that (D) also holds. Let $u=V f \in \mathcal{F}$ with $f \in L$ such that $\|u\|=\|f\|_{L}$. Define $v:=V|f| \in \mathcal{F}$. By nonnegativity of $V$, it follows that $|u| \leq v$. Moreover, $\|v\|=\|\pi(|f|)\|_{L / N} \leq\||f|\|_{L} \leq$ $\|f\|_{L}=\|u\|$.

Let $\left(\mathcal{F}^{*},\|\|\right)$ be the dual space with the dual norm, and let $\widetilde{V}^{*}: \mathcal{F}^{*} \rightarrow$ $(L / N)^{*}$ be the adjoint operator of $\widetilde{V}$. Then $\widetilde{V}^{*}$ is also an isometric isomorphism. Let $U: \mathcal{F}^{*} \rightarrow \mathcal{F}$ be the duality mappings of weight $\gamma^{*}$. Then

$$
U=\tilde{V} \circ \widetilde{I} \circ \widetilde{V}^{*}
$$

Indeed, for $\phi \in \mathcal{F}^{*}$ it holds that

$$
\begin{aligned}
\left\langle\phi,\left(\widetilde{V} \circ \widetilde{I} \circ \widetilde{V}^{*}\right) \phi\right\rangle & ={ }_{(L / N)^{*}}\left\langle\widetilde{V}^{*} \phi, \widetilde{I}\left(\widetilde{V}^{*} \phi\right)\right\rangle_{(L / N)} \\
& =\left\|\widetilde{V}^{*} \phi\right\|_{(L / N)^{*}}\left\|\widetilde{I}\left(\widetilde{V}^{*} \phi\right)\right\|_{(L / N)} \\
& =\left\|\widetilde{V}^{*} \phi\right\|_{(L / N)^{*}} \gamma^{*}\left(\left\|\widetilde{V}^{*} \phi\right\|_{(L / N)^{*}}\right)=\|\phi\| \gamma^{*}(\|\phi\|),
\end{aligned}
$$

and

$$
\left\|\left(\widetilde{V} \circ \widetilde{I} \circ \widetilde{V}^{*}\right) \phi\right\|=\left\|\widetilde{I}\left(\widetilde{V}^{*} \phi\right)\right\|_{(L / N)^{*}}=\gamma^{*}\left(\left\|\widetilde{V}^{*} \phi\right\|_{(L / N)^{*}}\right)=\gamma^{*}(\|\phi\|) .
$$

By uniqueness, (5.2) follows. Let $V^{*}: \mathcal{F}^{*} \rightarrow L^{*}$ be the adjoint of $V$. Then $V^{*}=\pi^{*} \circ \widetilde{V}^{*}$. It follows from (5.1) and (5.2) that

$$
U=V \circ I \circ V^{*}
$$


This representation of the duality mapping $U$ implies that the potentials in $\mathcal{F}$ are of the form

$$
U \phi=\left(V \circ I \circ V^{*}\right) \phi, \quad \text { for } \phi \in \mathcal{F}_{+}^{*} .
$$

Finally, we note that if $V: L \rightarrow \mathcal{G}$ is $1-1$, i.e., if $N=\{0\}$, there is no need to replace $L$ by the quotient space.

We now list some special cases that fit in the above framework.

(A1) This example is studied in [1], and generalizes the $L^{p}$-potential theory introduced in [21].

Let $X$ be a locally compact separable metric space, and let $(Y, \nu)$ be a measure space, where $\nu$ is a positive measure defined on Borel subsets of $Y$. We take $L=L^{p}(Y, \nu)$ where $1<p<\infty$. Let $v: X \times Y \rightarrow \mathbb{R}_{+}$be a kernel on $X \times Y$ such that $v(\cdot, y)$ is lower semicontinuous on $X$ for each $y \in Y$, and $v(x, \cdot)$ is measurable on $Y$ for each $x \in X$. For a nonnegative $f \in L^{p}(Y, \nu)$ let

$$
V f(x):=\int_{Y} v(x, y) f(y) \nu(d y)
$$

Let $\mathcal{N}$ be the smallest ideal containing the family of all sets $\{x \in X$ : $V|f|(x)=+\infty\}$ with $f \in L^{p}(Y, \nu)$. Then formula (5.5) defines $V f \mathcal{N}$ a.e. for every $f \in L^{p}(Y, \nu)$. Note that properties (i) and (ii) hold. Set $\mathcal{F}:=V\left(L^{p}(Y, \nu)\right)$ and $\|u\|=\|V f\|=\|f\|_{p}$ (where $f$ minimizes the quotient norm). Then $(\mathcal{F},\|\|)$ satisfies hypotheses $(\mathbf{F}),(\mathbf{C})$ and $(\mathbf{D})$.

Let $\gamma(t)=t^{p-1}$. The inverse of $\gamma$ is $\gamma^{*}(t)=t^{p^{\prime}-1}$ with $1 / p+1 / p^{\prime}=1$. The duality mapping $I: L^{p^{\prime}}(Y, \nu) \rightarrow L^{p}(Y, \nu)$ of weight $\gamma^{*}$ is of the form

$$
I g=|g|^{p^{\prime}-1} \operatorname{sign}(g) \text {, }
$$

with $g \in L^{p^{\prime}}(Y, \nu)$. This leads to the following representation of potentials: For $\phi \in \mathcal{F}_{+}^{*}$

$$
U \phi=V\left(\left|V^{*} \phi\right|^{p^{\prime}-1} \operatorname{sign}\left(V^{*} \phi\right)\right) .
$$

In particular, if $\mu$ is a Radon measure on $X$ such that $V^{*} \mu \in L^{p^{\prime}}(Y, \nu)$, where $V^{*} \mu(y):=\int_{X} v(x, y) \mu(d x)$, then $\mu$ defines a nonnegative, continuous linear functional on $\mathcal{F}$. The potential of $\mu$ is

$$
U \mu(x)=\int_{Y} v(x, y)\left(\int_{X} v(z, y) \mu(d z)\right)^{p^{\prime}-1} \nu(d y) .
$$


Special cases of the $L^{p}$-potential theory are obtained with $X=Y=\mathbb{R}^{N}$, $\nu$ the $N$-dimensional Lebesgue measure, and the Bessel kernel $G_{\alpha, p}, 0<\alpha$, or the Riesz kernel $I_{\alpha, p}, 0<\alpha<N$. These kernels define 1-1 operators. The detailed study of corresponding potential theories is given in [16].

(A2) This is studied in [18].

Let $X$ be a separable metric space, and let $m$ be a finite Borel measure on $X$. Let $\left(T_{t}, t \geq 0\right)$ be a strongly continuous contraction semigroup on $L^{p}(X, m)$, where $1<p<\infty$. For $\alpha>0$ let

$$
V_{\alpha}=\frac{1}{\Gamma(\alpha / 2)} \int_{0}^{\infty} t^{\alpha / 2-1} e^{-t} T_{t} d t
$$

be the gamma transform of the semigroup $\left(T_{t}\right)$. It is proved in [18, Proposition 2.3], that $V_{\alpha}$ is 1-1. Properties (i) and (ii) of $V_{\alpha}$ are easily checked. Define the Sobolev spaces $\left(\mathcal{F}_{\alpha, p},\|\|_{\alpha, p}\right)$ by $\mathcal{F}_{\alpha, p}=V_{\alpha}\left(L^{p}(X, m)\right),\|u\|_{\alpha, p}=$ $\|f\|_{p}$, where $u=V_{\alpha, p}, f \in L^{p}(X, m)$. For the ideal $\mathcal{N}$ we take sets of $m$ measure zero. The duality mapping $U: \mathcal{F}_{\alpha, p}^{*} \rightarrow \mathcal{F}_{\alpha, p}$ has the same form as in (5.6), for $\phi \in \mathcal{F}_{\alpha, p}^{*}$.

(A3) Besov spaces.

Let $X=\mathbb{R}^{N}, Y=\mathbb{R}^{N} \times \mathbb{N}$, and let $1<p<\infty, 1<q<\infty$ with conjugate exponents $p^{\prime}$ and $q^{\prime}$. We take $L=l^{q}\left(L^{p}\right)$ with the underlying Lebesgue measure. The $l^{q}\left(L^{p}\right)$-norm of $f: \mathbb{R}^{N} \times \mathbb{N} \rightarrow \mathbb{R}$ is given by

$$
\|f\|_{l^{q}\left(L^{p}\right)}:=\left(\sum_{n=1}^{\infty}\|f(\cdot, n)\|_{p}^{q}\right)^{1 / q} .
$$

Let $\eta_{0}=\eta \neq 0$ be a $C^{\infty}$-function supported in the unit ball. For $n \in \mathbb{N}$ let $\eta_{n}(x)=2^{n N} \eta\left(2^{n} x\right)$. For a nonnegative and measurable $f=\left(f_{n}\right)$ on $\mathbb{R}^{N} \times \mathbb{N}$, let

$$
V_{\alpha} f:=\sum_{n=0}^{\infty} 2^{-n \alpha} \eta_{n} * f_{n} .
$$

According to $\left[1\right.$, Theorem 4.1.7 and Proposition 4.4.1], $\mathcal{F}:=V_{\alpha}\left(l^{q}\left(L^{p}\right)\right)$ is equal to the Besov space $B_{\alpha}^{p, q}$. The norm on $\mathcal{F}$ is given by

$$
\|u\|=\left\|V_{\alpha} f\right\|=\|f\|_{l^{q}\left(L^{p}\right)} .
$$

where $f \in l^{q}\left(L^{p}\right)$ minimizes the quotient norm. Since properties (i), and (iii) are satisfied for $V_{\alpha}$, the Besov space $B_{\alpha}^{p, q}$ with the $l^{q}\left(L^{p}\right)$ quotient norm also fits in our general framework. 
Let $\gamma(t)=t^{q-1}$ with the inverse weight function $\gamma^{*}(t)=t^{q^{\prime}-1}$. The corresponding duality mapping $I: l^{q^{\prime}}\left(L^{p^{\prime}}\right) \rightarrow l^{q}\left(L^{p}\right)$ is of the form

$$
I g=\left\{\|g(\cdot, n)\|_{p^{\prime}}^{q^{\prime}-p^{\prime}}|g(\cdot, n)|^{p^{\prime}-1} \operatorname{sign}(g(\cdot, n)), n \in \mathbb{N}\right\},
$$

leading to a representation of a nonlinear potential of a positive measure $\mu$ on $\mathbb{N}$ given in $[1,(4.4 .9)]$.

The case studied in [22], similar to the described one, also satisfies our hypotheses.

(A4) Lizorkin-Triebel spaces.

The setting is as in (A3), with $l^{q}\left(L^{p}\right)$ spaces replaced by $L^{p}\left(l^{q}\right)$. According to $\left[1\right.$, Theorem 4.3 .10 and Proposition 4.4.1], $\mathcal{F}:=V_{\alpha}\left(L^{p}\left(l^{q}\right)\right)$ is equal to the Lizorkin-Tribel space $F_{\alpha}^{p, q}$.

(A5) Potential theory for Orlicz spaces.

Let $\Omega \subset \mathbb{R}^{N}$, and let $A$ and $A^{*}$ be complementary $N$-functions both satisfying the $\Delta_{2}$-condition (see [20] for details). Let $\left(L_{A}(\Omega),\|\|\|\|_{A}\right)$ be the Orlicz space with the Luxemburg norm. Then $L_{A}(\Omega)$ is reflexive, and the dual space may be identified with $L_{A^{*}}(\Omega)$. Moreover, by [20, Theorem 18.5], the Luxemburg norm is smooth, and therefore strictly convex by the duality argument. By taking $L=L_{A}(\Omega)$, and defining the operator $V$ with an appropriate kernel function $v$, this case fits in the general framework.

Potential theory for Orlicz spaces was studied in [2] and [3].

ExAmple B. Weighted Sobolev spaces $H^{1, p}(\Omega ; \mu)$ and $H_{0}^{1, p}(\Omega ; \mu)$.

We follow the presentation from [17]. Let $\Omega$ be an open subset in $\mathbb{R}^{N}$, $N \geq 2$, and let $1<p<\infty$. Let $w$ be a locally integrable function on $\mathbb{R}^{N}$, and let $d \mu(x)=w(x) d x$, where $d x$ is the Lebesgue measure on $\mathbb{R}^{N}$. We assume that $w$ is $p$-admissible (see [17, p. 7], for details). For a function $u \in C^{\infty}(\Omega)$ let

$$
\|u\|_{1, p}=\left(\int_{\Omega}|u|^{p} d \mu\right)^{1 / p}+\left(\int_{\Omega}\|\nabla u\|^{p} d \mu\right)^{1 / p} .
$$

The weighted Sobolev space $H^{1, p}(\Omega ; \mu)$ is defined to be the completion of $\left\{u \in C^{\infty}(\Omega):\|u\|_{1, p}<\infty\right\}$. The space $H_{0}^{1, p}(\Omega ; \mu)$ is the closure of $C_{0}^{\infty}(\Omega)$ in $H^{1, p}(\Omega ; \mu)([17$, p. 9$])$.

The Sobolev spaces $H^{1, p}(\Omega ; \mu)$ and $H_{0}^{1, p}(\Omega ; \mu)$ are uniformly convex, hence reflexive $([17$, p. 13$])$, and the norm \|\|$_{1, p}$ is smooth. Hence, hypotheses $(\mathbf{F})$ are satisfied. Let $\mathcal{N}$ be the family of all $\mu$-zero sets. Since 
convergence in the $L^{p}$-norm implies $\mu$-a.e. convergence of a subsequence, (C) holds. Moreover, since the contraction operates on $H^{1, p}(\Omega ; \mu)$ and $H_{0}^{1, p}(\Omega ; \mu)$, the hypothesis $(\mathbf{D})$ also holds true.

Our main interest will be in the Sobolev space $H_{0}^{1, p}(\Omega ; \mu)$ for bounded $\Omega$. In this case, it follows from the Poincaré inequality [17, p. 9 and Lemma 1.7], that

$$
\|u\|:=\left(\int_{\Omega}\|\nabla u\|^{p} d \mu\right)^{1 / p}
$$

defines an equivalent norm on $H_{0}^{1, p}(\Omega ; \mu)$. Let $(\mathcal{F},\|\|)$ denote the space $H_{0}^{1, p}(\Omega ; \mu)$ with the norm as in $(5.8)$. Then $(\mathcal{F},\|\|)$ also satisfies hypotheses $(\mathbf{F}),(\mathbf{C})$ and $(\mathbf{D})$. The duality mapping $J: \mathcal{F} \rightarrow \mathcal{F}^{*}$ is given by

$$
\langle J u, v\rangle=\int_{\Omega}|\nabla u|^{p-2} \nabla u \cdot \nabla v d \mu,
$$

for $u, v \in \mathcal{F}$. Hence $J$ may be identified with the weighted $p$-Laplacian

$$
\Delta_{p, w}=\operatorname{div}\left(w(x)|\nabla u|^{p-2} \nabla u\right)
$$

(see $[17$, p. 55 and p. 59]). Potentials are identified as supersolutions of the equation $-\Delta_{p, w}(u)=0$ in $\Omega$.

The following example is a case in point where $\left(\mathbf{D}^{+}\right)$is valid and not (D). $\mathbb{R}^{N}$.

Example C. The Sobolev space $H_{0}^{m, 2}(B)$ where $B$ is an open ball in

The space $H_{0}^{m, 2}(B)$ is the closure of $C_{0}^{\infty}(B)$ with respect to the norm $\|u\|_{m, 2}=\sum_{|\alpha| \leq m}\left\|D^{\alpha}(u)\right\|_{2}$. By the Poincaré inequality,

$$
\|u\|:=\left(\sum_{|\alpha|=m}\left\|D^{\alpha}(u)\right\|_{2}^{2}\right)^{1 / 2}
$$

defines an equivalent norm on $H_{0}^{m, 2}(B)$. The space $\left(H_{0}^{m, 2}(B),\|\|\right)$ is reflexive, strictly convex and smooth. Hence, hypotheses $(\mathbf{F})$ are satisfied, and clearly, (C) hold as well. We check that $\left(\mathbf{D}^{+}\right)$also holds. It is easy to see that or $u, v \in H_{0}^{m, 2}(B)$

$$
\frac{d}{d t}\|u+t v\|_{\mid t=0}^{2}=2 \sum_{|\alpha|=m} \int_{B}\left(D^{\alpha} u\right)\left(D^{\alpha} v\right)=2 \int_{B}(-\Delta)^{m} u v .
$$


Let $J$ be the duality mapping with weight $\gamma(t)=t$. Then from the above inequality and (2.2),

$$
\langle J u, v\rangle=\sum_{|\alpha|=m} \int_{B}\left(D^{\alpha} u\right)\left(D^{\alpha}(v)\right)=\int_{B}(-\Delta)^{m} u v .
$$

so $J$ may be identified with $(-\Delta)^{m}$. Let $\phi \in H_{0}^{m, 2}(B)^{*}$. Then $\phi=J u$ for a $u \in H_{0}^{m, 2}(B)$. Suppose that $\phi$ is nonnegative, i.e., $J u \geq 0$ in $H_{0}^{m, 2}(B)^{*}$. Then $(-\Delta)^{m} u \geq 0$ in the distributional sense. From [4, Theorem 1], it follows that $u \geq 0$ on $B$. Hence $u=U \phi \geq 0$, i.e., $U$ is nonnegative. By Proposition 2.2 , this is equivalent to $\left(\mathbf{D}^{+}\right)$.

\section{REFERENCES}

[1] D. R. Adams and L. I. Hedberg, Function Spaces and Potential Theory, Springer, 1996.

[2] N. Aïssaoui and A. Benkirane, Capacités dans les espaces d'Orlicz, Ann. Sci. Math. Québec, 18 (1994), 1-23.

[3] — Potentiel non linéaire dans les espaces d'Orlicz, Ann. Sci. Math. Québec, 18 (1994), 105-118.

[4] A. Ancona, Une propriété des espaces de Sobolev, C. R. Acad. Sci. Paris, Sèr. I, 292 (1981), 477-480.

[5] N. Aronszajn and K. T. Smith, Functional spaces and functional completion, Ann. Inst. Fourier (Grenoble), 13 (1956), no. 2, 125-185.

[6] E. Asplund, Positivity of duality mappings, Bull. Amer. Math. Soc., 73 (1967), 200-203.

[7] H. Attouch and C. Picard, Problemes variationnels et theorie du potentiel non lineaire, Annales Fac. Sciences Toulouse, 1 (1979), no. 2, 89-136.

[8] I. Cioranescu, Geometry of Banach Spaces, Duality Mappings and Nonlinear Problems, Kluwer, 1990.

[9] C. V. Coffman and C. L. Grover, Obtuse cones in Hilbert spaces and applications to partial differential equations, Journal of Functional Analysis, 35 (1980), 369-396.

[10] J. Diestel, Geometry of Banach Spaces - Selected Topics, Lecture Notes in Mathematics 485, Springer, 1975.

[11] D. Feyel and A. de La Pradelle, Topologies fines et compactifications assciées a certains espaces de Dirichlet, Ann. Inst. Fourier (Grenoble), 27 (1977), no. 4, 121-146.

[12] P. A. Fowler, Potential Theory in Banach spaces of functions, A Condenser Theorem, J. Math. Anal. Appl., 33 (1971), no. 2, 310-322.

[13] B. Fuglede, Applications du théorème du minimax à l'étude de diverses capacités, C. R. Acad. Sci. Paris Sér. A-B, 266 (1968), 921-923.

[14] M. Fukushima, Two topics related to Dirichlet forms: quasi everywhere convergence and additive functionals, Dirichlet Forms, Lecture Notes in Mathematics 1563 (G. Dell'Antonio and U. Mosco, eds.), Springer (1993). 
[15] M. Fukushima and H. Kaneko, On $(r, p)$-capacities for general Markovian semigroups, Infinite dimensional analysis and stochastic processes (S. Albeverio, ed.), Pitman (1985).

[16] V. P. Havin and V. G. Maz'ya, Non-linear potential theory, Russian Math. Surveys, 27 (1972), no. 6, 71-148.

[17] J. Heinonen, T. Kilpeläinen and O. Martio, Nonlinear Potential Theory of Degenerate Elliptic Equations, Oxford University press, Oxford, 1993.

[18] T. Kazumi and I. Shikegawa, Measures of finite $(r, p)$-energy and potentials on a separable metric space, Séminaire de Probabilités, XXVI (J. Azéma and M. Yor, eds.), Lecture Notes in Mathematics 1526, Springer (1992), pp. 415-444.

[19] N. Kenmochi and Y. Mizuta, The gradient of a convex function on a regular functional space and its potential theoretic properties, Hiroshima Math. J., 4 (1974), 743-763.

[20] M. A. Krasnosel'skii and Ya. B. Rutickii, Convex Functions and Orlicz Spaces, P. Noordhoff, 1961.

[21] N. G. Meyers, A theory of capacities for potentials of functions in Lebesgue classes, Math. Scand., 26 (1970), 255-292.

[22] M. Rao and J. Sokolowski, Non-linear Balayage and Applications, Illinois J. Math., 44 (2000), 310-328.

Murali Rao

Department of Mathematics

University of Florida

358 Little Hall

Gainesville, Fl 32611-8105

U.S.A.

rao@math.ufl.edu

Zoran Vondraček

Department of Mathematics

University of Zagreb

Bijenička c. 30

10000 Zagreb

Croatia

vondra@math.hr 